\title{
$\left.{ }_{S S C I E N C E}^{\{M A R I X}\right\}$ Mascot Search Results
}

\section{Protein View}

Match to: Plasmodium falciparum 3D7|MAL10|PF10 0155|Pf Score: 4921 Annotation |Plasmodium falciparum TIGR/ (protein coding) enolase

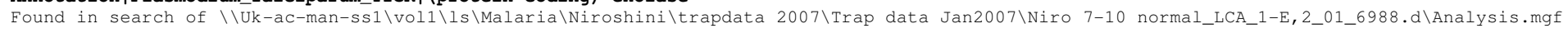
Nominal mass $\left(\mathrm{M}_{\mathrm{r}}\right): \mathbf{4 8 6 4 7}$; Calculated $\mathrm{pI}$ value: $\mathbf{6 . 2 1}$ NCBI BLAST search of Plasmodium_falciparum_3D7|MAL10|PF10 0155|Pf against nr Unformatted sequence string for pasting into other applications

Variable modifications: Carbamidomethyl (C), Oxidation (M), Heavy IsoLeu

Cleavage by Trypsin: cuts C-term side of KR unless next residue is

Sequence Coverage: $52 \%$

Matched peptides shown in Bold Red

1 MAHVITRINA REILDSRGNP TVEVDLETNL GIFRAAVPSG ASTGIYEALE 51 LRDNDKSRYL GKGVOKAIKN INEIIAPKLI GMNCTEOKKI DNLMVEELDG 101 SKNEWGWSKS KLGANAILAI SMAVCRAGAA ANKVSLYKYL AQLAGKKSDO 151 MVLPVPCLNV INGGSHAGNK LSFOEFMIVP VGAPSFKEAL RYGAEVYHTI 201 KSEIKKKYGI DATNVGDEGG FAPNILNANE ALDLLVTAIK SAGYEGKVKI 251 AMDVAASEFY NSENKTYDLD FKTPNNDKSL VKTGAOLVDL YIDLVKKYPI 301 VSIEDPFDOD DWENYAKLTA AIGKDVOIVG DDLLVTNPTR ITKALEKNAC 351 NALLLKVNQI GSITEAIEAC LLSOKNNWGV MVSHRSGETE DVFIADLVVA 401 LRTGQIKTGA PCRSERNAKY NQLLRIEESI GNNAVFAGEK FRLQLN

Show predicted peptides also

Sort Peptides By $\bigcirc$ Residue Number $\bigcirc$ Increasing Mass $\bigcirc$ Decreasing Mass

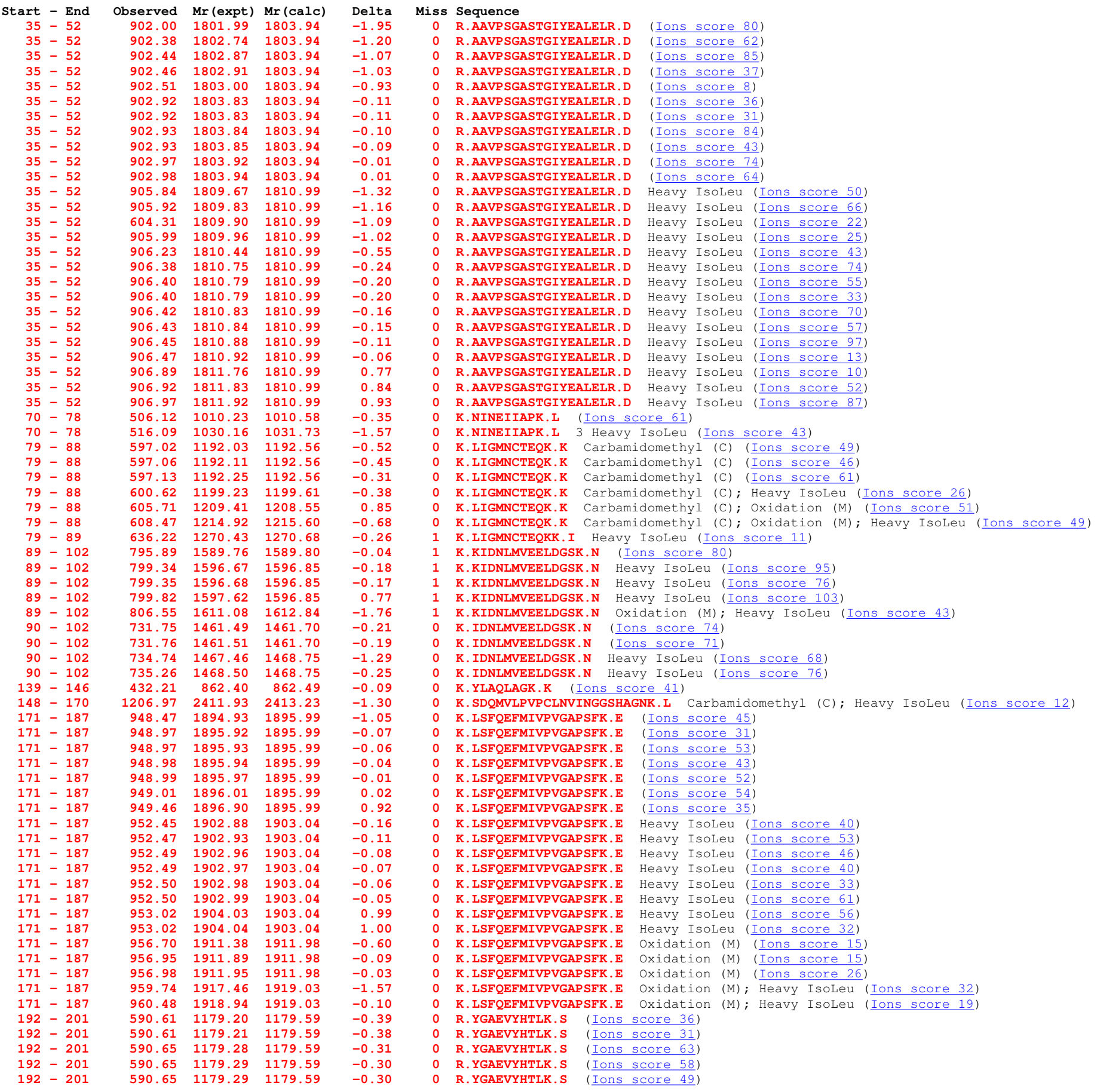

file://S:IMalaria\John\JEH Zoom paper|Zoom paper suooporting info\Appendix 4\SHMT and Enol... 03/05/2007 
250 - 26

$250-265$
$250-265$

$250-265$

$250-265$

$250-265$
$250-265$

250 - 265

$250-265$

$250-265$

$250-265$

$250-265$

$250-265$

$250-265$

$250-265$

$250-265$

$250-265$
$250-265$

$266-272$

$283-296$

$283-296$

283 - 296

$283-296$
283 - 296

$283-296$

$283-296$

$283-296$

$283-296$

$283-296$

$283-296$

283 - 296
283 - 296

$283-296$

$283-296$

283 - 296
283 - 296

$283-296$

$297-317$

297 - 317

$297-317$

297 - 317

$297-317$
$297-317$

$297-317$

$297-317$
$297-317$

$297-317$

297 - 317

$298-317$

$298-317$
$298-317$

$298-317$

$298-317$

$298-317$
$298-317$

$298-317$

$325-340$

$325-340$

$325-340$

$325-340$
$325-340$

$325-340$
$325-340$

$325-340$

$325-340$
$325-340$

325 - 340

325 - 340

$325-340$

$325-340$

$325-340$

$325-340$

$325-340$
$325-340$

325 - 340

$348-356$

357 - 375

$357-375$

$357-375$

$357-375$
$357-375$

357 - 375

$357-375$
$357-375$

357 - 375

$357-375$

357 - 375

357 - 375

$357-375$

$357-375$
$357-375$

357 - 375

$357-375$
$357-375$

$357-375$

$357-375$

$357-375$

357 - 375

$357-375$

$357-375$
$357-375$

$357-375$

$357-375$

$357-375$

$357-375$
$357-375$

$357-375$ $\begin{array}{llll}893.92 & 1785.83 & 1787.80\end{array}$ $\begin{array}{llll}94.35 & 1786.69 & 1787.80\end{array}$ $\begin{array}{lll}94.39 & 1786.76 & 1787.80\end{array}$ $\begin{array}{lll}894.86 & 1787.70 & 1787.80\end{array}$ $\begin{array}{lll}894.87 & 1787.72 & 1787.80\end{array}$ $\begin{array}{lll}894.88 & 1787.74 & 1787.80\end{array}$ $\begin{array}{lll}894.88 & 1787.76 & 1787.80\end{array}$ $\begin{array}{lll}894.91 & 1787.80 & 1787.80\end{array}$ $\begin{array}{lll}895.25 & 1788.48 & 1787.80\end{array}$ $\begin{array}{lll}95.42 & 1788.82 & 1787.80\end{array}$ $\begin{array}{lll}897.84 & 1793.66 & 1794.85\end{array}$ $\begin{array}{lll}998.36 & 1794.71 & 1794.85\end{array}$ $898.39 \quad 1794.77 \quad 1794.85$ $\begin{array}{lll}898.42 & 1794.84 & 1794.85\end{array}$ $\begin{array}{lll}898.47 & 1794.92 & 1794.85\end{array}$ $\begin{array}{lll}902.85 & 1803.69 & 1803.80\end{array}$ $\begin{array}{lll}902.86 & 1803.71 & 1803.80\end{array}$ $\begin{array}{lll}906.00 & 1809.98 & 1810.85\end{array}$ $\begin{array}{lll}906.32 & 1810.63 & 1810.85\end{array}$ $\begin{array}{lrr}51.19 & 900.36 & 900.42\end{array}$ $\begin{array}{lll}74.32 & 1546.63 & 1546.86\end{array}$ $\begin{array}{lll}74.34 & 1546.66 & 1546.86\end{array}$ $774.36 \quad 1546.70 \quad 1546.86$ $\begin{array}{lll}74.36 & 1546.70 & 1546.86\end{array}$ $\begin{array}{lll}774.36 & 1546.71 & 1546.86\end{array}$ $\begin{array}{lll}774.39 & 1546.77 & 1546.86\end{array}$ $\begin{array}{lll}774.45 & 1546.89 & 1546.86\end{array}$ $\begin{array}{lll}774.87 & 1547.73 & 1546.86\end{array}$ $\begin{array}{lll}777.36 & 1552.71 & 1553.91\end{array}$ $\begin{array}{lll}77.77 & 1553.54 & 1553.91\end{array}$ $777.83 \quad 1553.65 \quad 1553.91$ $\begin{array}{lll}777.84 & 1553.66 & 1553.91\end{array}$ $\begin{array}{lll}777.86 & 1553.71 & 1553.91\end{array}$ $\begin{array}{lll}777.88 & 1553.75 & 1553.91\end{array}$ $\begin{array}{lll}777.90 & 1553.79 & 1553.91\end{array}$ $777.96 \quad 1553.91 \quad 1553.91$ $\begin{array}{lll}857.96 & 2570.86 & 2571.18\end{array}$ $\begin{array}{lll}886.49 & 2570.97 \quad 2571.18\end{array}$ $\begin{array}{lll}886.52 & 2571.02 \quad 2571.18\end{array}$ $858.04 \quad 2571.11 \quad 2571.18$ $\begin{array}{llll}858.13 & 2571.36 & 2571.18\end{array}$ $\begin{array}{lll}1286.99 & 2571.96 & 2571.18\end{array}$ $\begin{array}{lll}858.70 & 2573.06 & 2571.18\end{array}$ $\begin{array}{lll}1293.48 & 2584.95 & 2585.28 \\ 1293.57 & 2585.13 & 2585.28\end{array}$ $\begin{array}{llll}862.74 & 2585.20 & 2585.28\end{array}$ $862.75 \quad 2585.22 \quad 2585.28$ $\begin{array}{lll}863.02 & 2586.04 & 2585.28\end{array}$ $\begin{array}{lll}2442.84 & 2443.09\end{array}$ $\begin{array}{lll}222.45 & 2442.89 & 2443.09\end{array}$ $\begin{array}{lll}1222.45 & 2442.90 & 2443.09\end{array}$ $1222.87 \quad 2443.73 \quad 2443.09$ $\begin{array}{lll}1223.43 & 2444.84 & 2443.09\end{array}$ $\begin{array}{lll}1229.00 & 2455.98 & 2457.19\end{array}$ $\begin{array}{lll}1229.45 & 2456.89 & 2457.19\end{array}$ $\begin{array}{lll}229.46 & 2456.90 & 2457.19\end{array}$ $\begin{array}{lll}877.17 & 1752.33 & 1753.92 \\ 877.45 & 1752.88 & 1753.92\end{array}$ $\begin{array}{lll}877.88 & 1753.75 & 1753.92\end{array}$ $\begin{array}{lll}77.92 & 1753.83 & 1753.92\end{array}$ $\begin{array}{lll}877.93 & 1753.84 & 1753.92\end{array}$ $877.93 \quad 1753.84 \quad 1753.92$ $\begin{array}{lll}877.94 & 1753.86 & 1753.92\end{array}$ $\begin{array}{lll}877.98 & 1753.95 & 1753.92\end{array}$ $\begin{array}{llll}878.395 & 1754.76 & 1753.92\end{array}$ $\begin{array}{lll}880.85 & 1759.69 & 1760.97 \\ 880.91 & 1759.81 & 1760.97\end{array}$ $\begin{array}{lll}881.40 & 1760.79 & 1760.97\end{array}$ $\begin{array}{lll}881.41 & 1760.82 & 1760.97\end{array}$ $\begin{array}{lll}881.42 & 1760.83 & 1760.97\end{array}$ $881.82 \quad 1761.62 \quad 1760.97$ $\begin{array}{lll}881.82 & 1761.63 & 1760.97\end{array}$ $\begin{array}{lll}881.86 & 1761.71 & 1760.97\end{array}$ $\begin{array}{llll}881.91 & 1761.82 & 1760.97\end{array}$ $\begin{array}{lll}81.92 & 1761.83 & 1760.97\end{array}$ $\begin{array}{lll}508.71 & 1015.41 & 1015.55\end{array}$ $\begin{array}{lll}1036.65 & 2071.29 & 2073.08\end{array}$ $\begin{array}{lll}1036.98 & 2071.95 & 2073.08\end{array}$ $\begin{array}{lll}1037.44 & 2072.86 & 2073.08\end{array}$ $\begin{array}{lll}691.96 & 2072.87 \quad 2073.08\end{array}$ $\begin{array}{lll}1037.45 & 2072.89 & 2073.08\end{array}$ $\begin{array}{lll}1037.46 & 2072.91 & 2073.08\end{array}$ $1037.48 \quad 2072.95 \quad 2073.08$ $\begin{array}{lll}691.99 & 2072.95 & 2073.08\end{array}$ $1037.49 \quad 2072.96 \quad 2073.08$ $\begin{array}{lll}037.49 & 2072.96 & 2073.08\end{array}$ $\begin{array}{lll}1037.49 & 2072.97 & 2073.08\end{array}$ $\begin{array}{lll}037.49 & 2072.98 & 2073.08\end{array}$ $\begin{array}{lll}692.00 & 2072.98 \quad 2073.08\end{array}$ $\begin{array}{lll}637.50 & 2072.99 & 2073.08\end{array}$ $\begin{array}{lll}692.01 & 2073.02 & 2073.08\end{array}$ $\begin{array}{lll}692.02 & 2073.04 & 2073.08\end{array}$ $\begin{array}{lll}1037.54 & 2073.06 & 2073.08 \\ 1037.59 & 2073.17 & 2073.08\end{array}$ $\begin{array}{lll}692.17 & 2073.49 & 2073.08\end{array}$ $\begin{array}{lll}1044.53 & 2087.05 & 2087.18\end{array}$ $\begin{array}{lll}1047.31 & 2092.60 & 2094.23\end{array}$ $1047.48 \quad 2092.94 \quad 2094.23$ $\begin{array}{lll}1047.52 & 2093.02 & 2094.23\end{array}$ $\begin{array}{lll}1047.54 & 2093.06 & 2094.23\end{array}$ $\begin{array}{lll}698.91 & 2093.72 & 2094.23\end{array}$ $\begin{array}{lll}698.95 & 2093.84 & 2094.23\end{array}$ $\begin{array}{lll}1047.97 & 2093.93 & 2094.23\end{array}$ $698.99 \quad 2093.94 \quad 2094.23$ $\begin{array}{lll}1047.98 & 2093.95 & 2094.23\end{array}$ $\begin{array}{lll}1048.00 & 2093.99 & 2094.23 \\ 1048.00 & 2093.99 & 2094.23\end{array}$
$-1.97$

$-1.12$

$-1.05$

$-0.09$

$-0.06$

$-0.05$

0.68

$-1.20$

$-0.14$

$-0.09$

0.07

$-0.11$

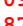

$-0.22$

$-0.23$

$-0.16$

$-0.16$

$-0.09$

0.02

0.87

$-1.20$

-0.38
-0.26

0.26

$-0.20$

$-0.13$

$-0.06$

$-0.01$

$-0.32$

$-0.22$

$-0.07$

0.18
0.78

1.88

$-0.33$

0.15

$-0.08$

0.76

$-0.25$

-0.20
-0.19

0.64

1. 75

$-0.30$

$-0.29$

$-1.59$

$-1.05$

-0.17
-0.10

0.08

$-0.08$

$-0.06$

0.84

0.84

$-1.16$

$-0.18$

$-0.16$

-0.14
0.65

0.66

0.74

0.86

0.86
-0.14

0.14
-1.79

$-1.13$

$-0.22$

$-0.19$

$-0.17$

$-0.13$

$-0.12$

0.12

$-0.12$

$-0.10$

-0.10
-0.08

$-0.06$

$-0.04$

0.09

0.42

$-0.13$

$-1.63$

$-1.29$

$-1.17$

$-0.51$

$-0.30$

$-0.29$

$-0.29$

$-0.24$

-0.24
-0.24

K. IAMDVAASEFYNSENK. T

0 K. IAMDVAASEFYNSENK. T

K. IAMDVAASEFYNSENK.

K. IAMDVAASEFYNSENK. I

K. IAMDVAASEFYNSENK. T

K. IAMDVAASEFYNSENK. T

K. IAMDVAASEFYNSENK.T

K. IAMDVAASEFYNSENK.T

K. IAMDVAASEFYNSENK.

K. IAMDVAASEFYNSENK.

$K$. IAMDVAASEFYNSENK. I

K. IAMDVAASEFYNSENK. $T$

K. IAMDVAASEFYNSENK. I

K. IAMDVAASEFYNSENK. T

Oxidation (M) (Ions score 76)

Oxidation (M) (Ions score 61 


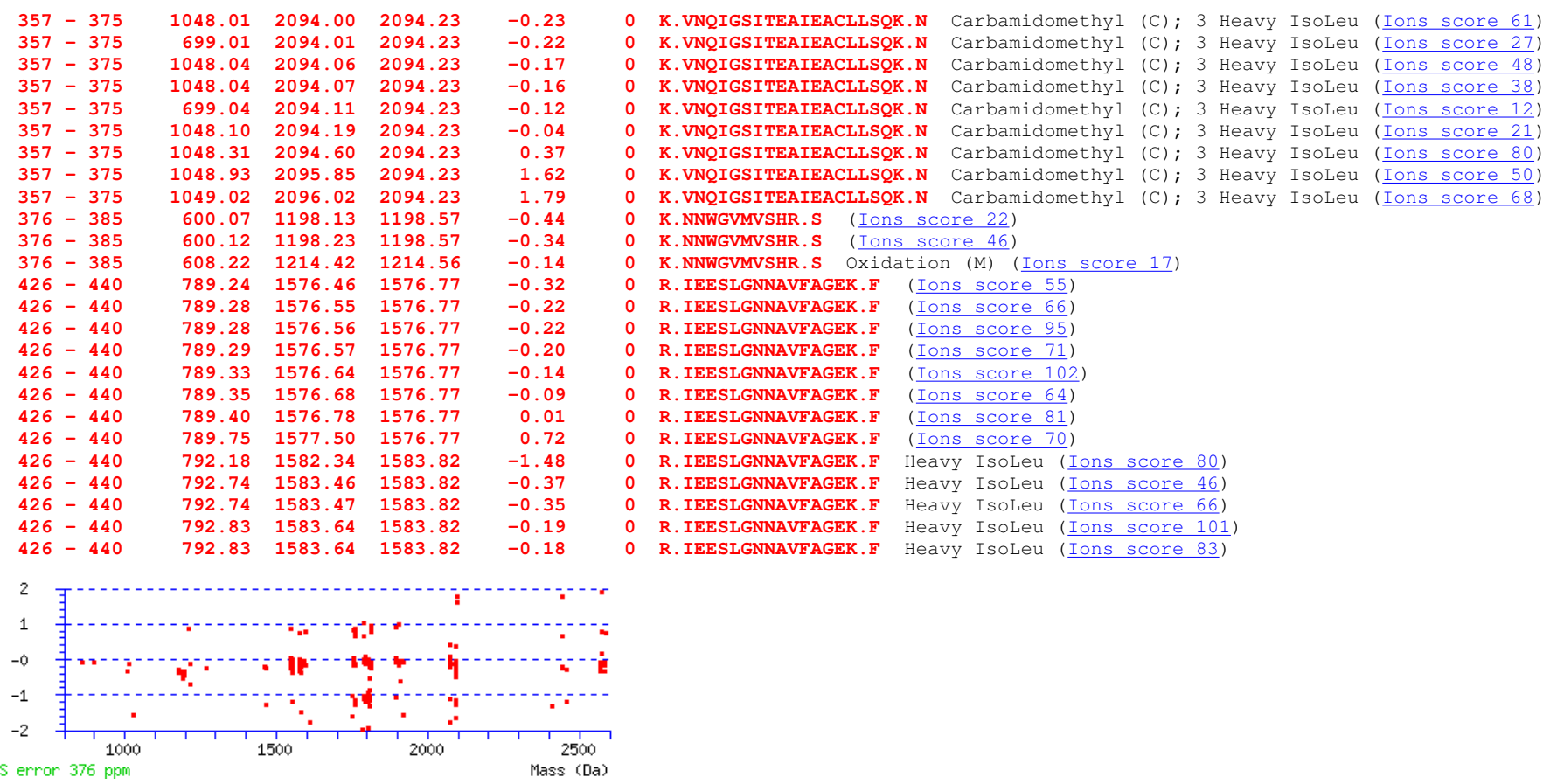

0 K.VNQIGSITEAIEACLLSOK.N Carbamidomethyl (C); 3 Heavy Isoleu (Ions score 61)

K. NQIGSITEAIEACLLSQK.N Carbamidomethy (C); 3 Heavy ISOLeu ( 1 ons score 27)

K.VNOIGSITEAIEACLISOK.N Carbamidomethyl (C); 3 Heavy IsoLeu (Ions score 38)

K.VNQIGSITEAIEACLLSOK.N Carbamidomethyl (C); 3 Heavy IsoLeu (Ions score 12)

K.VNOIGSITEAIEACLISOK.N Carbamidomethyl (C); 3 Heavy Isoleu (Ions score 80)

K.VNOIGSITEAIEACLLSOK.N Carbamidomethyl (C); 3 Heavy Isoleu (Ions score 50)

K.NNWGVMVSHR.S Oxidation (M) (Ions score 17 )

file://S:\Malaria\John\JEH Zoom paper\Zoom paper suooporting info\Appendix 4ISHMT and Enol... 03/05/2007 